\title{
Edge Detection Techniques for Quantifying Spatial Imaging System Performance and Image Quality
}

\author{
Oliver van \\ Zwanenberg \\ University of \\ Westminster \\ 115 New Cavendish St \\ London W1W 6UW, \\ United Kingdom \\ o.vanzwanenberg@my .
westminster.ac.uk
}

\author{
Sophie \\ Triantaphillidou \\ University of \\ Westminster \\ 115 New Cavendish St \\ triants@westminster. \\ ac.uk
}

\author{
Robin Jenkin \\ University of \\ Westminster \\ 115 New Cavendish St \\ $r \cdot$ jenkin@westminster
.ac.uk
}

\author{
Alexandra Psarrou
}

University of

Westminster

115 New Cavendish St

psarroa@westminster. ac.uk

\begin{abstract}
Measuring camera system performance and associating it directly to image quality is very relevant, whether images are aimed for viewing, or as input to machine learning and automated recognition algorithms. The Modulation Transfer Function (MTF) is a wellestablished measure for evaluating this performance. This study proposes a novel methodology for measuring system MTFs directly from natural scenes, by adapting the standardized Slanted Edge Method (ISO 12233). The method involves edge detection techniques, to select and extract suitable step edges from pictorial images. The scene MTF aims to account for camera non-linear scene dependent processes. This measure is more relevant to image quality modelling than the traditionally measured MTFs. Preliminary research results indicate that the proposed method can provide reliable MTFs, following the trends of the ISO 12233. Further development and validation are required before it is proposed as a universal camera measuring technique.
\end{abstract}

\section{Introduction}

Traditionally imaging system performance evaluation measures, such as the Modulation Transfer Function (MTF) (measure of signal transfer) and the Noise Power Spectrum (NPS) (measure of noise), are routinely taken from dedicated test charts, captured in controlled laboratory conditions. Extensive studies have been conducted to refine these measures that have resulted in International Standards publications, [1,2]. Recent work $[3,4]$, has explored the concept of deriving MTFs from images of pictorial scenes, using the noise measuring method (see section 1.2). This opens up the possibility for scene dependent performance measures, suitable for evaluating non-linear imaging processes incorporated in modern camera systems. Current unpublished studies are pushing this a step further, through exploring scene dependent image quality metrics. These metrics rely on input parameters with a physical basis, such as scene dependent MTFs/NPSs.

The aim of this paper is to introduce a novel methodology to measure capture MTFs directly from pictorial natural scenes, using the edge MTF method (section 1.2) and automatic edge extraction.

Rapid development in processing power in recent years has led to the use of neural networks and other computational techniques to be sought as solutions to previously intractable computer vision applications, such as autonomous driving. And while early results are impressive, autonomous driving will arguably represent the first mass deployment of computer vision into a safety critical system which will eventually be used by millions of people on a daily basis.

Computational image quality metrics, i.e. metrics that derive image quality based solely on image data resulting from captured/processed individual natural scenes, do an excellent job of providing guidance that correlates well with perceptual image quality and are impressive in their performance. A drawback, however, is that they do not provide any engineering insight, as the metrics does not relate to any physical parameters of the imaging system itself. Engineering based quality metrics on the other hand use imaging system parameters but have the drawback of not taking into account individual scene information. By attempting to estimate MTFs directly from captured natural scenes, it is hoped that intelligent diagnostics, based on the physics of the imaging system as well as the individual scene properties will be facilitated, providing system architects with additional tools with which to improve overall reliability of vision systems.

This paper will explore briefly the background of the Computational Image Quality Metrics (1.1) and Spatial Imaging Performance Measures (1.2), elaborating on how a performance measure derived from natural scenes may yield a more relevant input to image quality metrics, relevant to systems engineering. Subsequent sections will examine the proposed measurement framework (2), the 
parameters used (3) and present results from the preliminary studies $(4,5)$.

\subsection{Computational Image Quality Metrics}

Image quality modelling is studied by, and involves, a number of research areas: human cognition, visual psychophysics, computer vision, machine learning, signal/image processing and imaging systems engineering. Specialists have approached the development of image quality models from different perspectives and thus a broad spectrum of models/metrics has evolved, with various advantages and applications, [5]. Computational metrics have their origins in electronic engineering and computer vision research. Generally, the mechanics of computational image quality models rely on image data, after capture, and most often before display (with exception of a number of fidelity models which account for the display and viewing conditions) [6]. Also, (but not routinely) these models rely on input parameters that relate to human visual system characteristics, and/or cognition. They can be divided broadly into three categories, depending on whether information is available from an original image. Full-reference models have access to a reference image as well as test images. The most prominent are the methods that consider the sensitivity of the human visual system to structural and/or color information [7, 8]. Reduced reference models have access to a reduced set of features from the reference image $[9,10,11]$. Noreference models use information only from the test images with most successful approaches using natural scene statistics $[10,12]$. The appropriateness of each of these methods depends on the application.

Despite the success of some advanced computational image quality models in predicting visual quality, often with excellent accuracy, computational models in general do not consider the physics, design, engineering and performance characteristics of the imaging systems for capture and/or display of digital images [5]. This implies that, in such metrics, the perceived image quality is not considered as a function of the relevant imaging system. As a result, systematic changes in metric input parameters do not directly relate to changes in imaging system characteristics, making such metrics less relevant to system design and engineering.

On the other hand, engineering based quality/performance metrics, employ imaging system parameters as model parameters $[1,2,13]$, but fail to account for the varied characteristics of the captured natural scenes. Input parameters in engineering type of quality models are typically measures (spatial, color, noise, etc.) taken from carefully designed test charts, captured/measured under strict laboratory conditions.

An example of such a spatial model is the Acutance metric, $Q$, proposed by the IEEE standard for Camera Phone Image Quality [14].

$$
Q=\frac{\int_{0}^{v_{c u t}} \operatorname{SFR}(u) M(u) \operatorname{CSF}(u) d u}{\int_{0}^{\infty} \operatorname{CSF}(u) d u}
$$

Equation 1

where $u$ is the spatial frequency, $S F R$ is the imaging system performance measure (in this case the ISO 12233 Spatial Frequency Response (SFR) [1]), $M$ is the MTF for the medium in which the image is being viewed, i.e. a display or print, and CSF is the Contrast Sensitivity Function, a visual sensitivity model [15]. Amongst other image quality models that employ the MTF as an input parameter are the SQRI [16], EPIC [17].

Models of the human visual system and cognition may, or may not be accounted for, depending on the complexity of the metric. Quality predictions are as a result system dependent, which is desirable by engineers, but scene content independent. Yet, the most advanced algorithms (e.g. de-noising [18, 19], sharpening [20, 21]) incorporated in commercial camera systems are nonlinear, scene content aware; hence the introduction of the dead leaves MTF (based upon the noise MTF method, section 1.2) - an attempt to measure MTFs from a test chart that has a frequency spectrum resembling that of a natural scene [22].

An image quality framework, employing imaging system performance measures taken directly from natural scenes, thus avoiding test charts and accounting for scene content, has been recently proposed [5]. The same authors have been involved in implementing parts of the framework by measuring NPSs (noise) and MTFs (signal transfer) from captured images of natural scenes [4], but not directly from live scenes.

In this paper, we propose a method for deriving live capture system MTFs from captured natural scenes, using selected edge extraction and processing. The advantages of this approach are numerous: MTFs can be derived from cameras installed in real environments, for example CCTV or automotive. These measures would be representative of the capturing system performance as well as the system and scene content interactions. Importantly, scene dependent MTFs are expected to be more suitable input parameters to engineering image quality metrics, eliminating the weakness of such models of being generic and thus providing better correlations with observer quality scores. Research carried out in our labs indicates the potential merits of replacing traditional MTF and NPS measures in engineering image quality metrics with scene dependent versions of them $[4,5]$.

\subsection{Modulation Transfer Function}

The MTF, and the comparable SFR, is a wellestablished performance measure for optical and camera systems [23]. It provides imaging performance information in terms of sharpness and resolution, by quantifying the change in signal modulation from input 
to output, with respect to spatial frequency. MTF has its basis in Linear System Theory; in one-dimension it is defined as the modulus of the Fourier Transform of the system's Line Spread Function (LSF), LSF $(x)$ :

$$
\operatorname{MTF}(u)=\left|\int_{-\infty}^{+\infty} \operatorname{LSF}(x) e^{-2 \pi i u x} d x\right|
$$

Equation 2

The LSF is a measure of signal spread caused mainly by lens diffraction, optics, and image processing. It is the first derivative of the Edge Spread Function (ESF) i.e. the $1 \mathrm{D}$ intensity profile of a capture of a 'perfect edge', that is, an edge with spatial frequency content constant and equal to 1.0 over the system's bandwidth.

Capture system MTFs are measured using three different methods: sine-waves, edges, and noise [23]. For each method a variety of implementations have been proposed that tackle problems associated with discrete, often non-linear systems. All methods and implementations rely on capturing relevant test charts with specific features, under strict laboratory conditions.

A variation of the edge technique is proposed in ISO 12233 specifically for digital imaging systems - the slanted edge SFR. It employs slanted edge charts, as seen in Figure 1, including a number of slanted 'perfect edges' designed to avoid aliasing in the measurements. This method has been universally accepted as a reliable approach to estimate the MTF of discrete imaging systems, being developed and improved over the last two decades. Briefly, the following steps are involved in the slanted edge SFR measurement $[1,24,25]$ :

1. A chart, including at least one grayscale 'perfect' step edge (Figure 1) is photographed, with the edge captured at a tilted angle.

2. Pre-processing includes image linearization with respect to input luminance and edge selection as the Region of Interest (ROI).

3. Each row across the edge spread is an estimate of the system ESF. Super-sampling and averaging of the ESFs form the re-quantized (super-sampled) ESF.

4. The super-sampled ESF is differentiated and windowed to obtain a super-sampled LSF.

5. The normalized modulus of the Discrete Fourier Transform (DFT) returns the system SFR up to $4 \mathrm{x}$ the Nyquist limit (Equation 2).

\section{Framework}

The proposed framework aims to substitute the captured edge test chart in the standard ISO 12233 slanted edge method with any suitable captured scene (any suitable image) for deriving the given capturing system MTF directly from this scene. The basis of the edge MTF method, as discussed previously, is the use of a 'perfect edge' input to the ISO 12233 algorithm, specifically an edge with a step profile. We have developed a framework for automated detection, isolation and treatment of

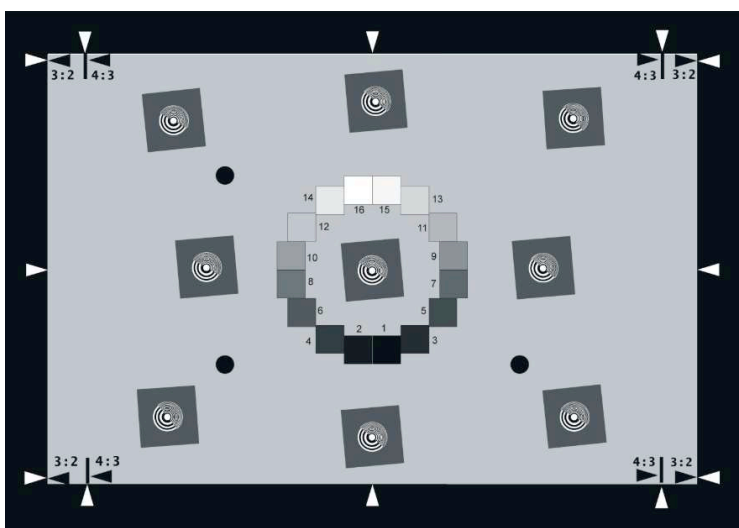

Figure 1: ISO 12233 SFR Test Chart

specific edges from any given natural scene. These selected and treated edges then become the input to the ISO 12233 algorithm (stages 1, 2), whilst stages 3-5 follow as before.

The detection of the edges is the first process applied in the framework, shown in Figure 2. We have considered here two edge detection methods, the first being the Canny approach [26], and the second a matched filter [27, 28].

Canny edge detection does not explicitly target step edges but instead detects changes in gradients [26]. Therefore, some parameter tuning and logic is needed to isolate the specific input profile required in the ROI. For example, a gradient is examined for each flagged edge to ensure there is only a single edge (no double edges). All relevant parameters are discussed in Section 3. The matched filter uses a convolution kernel to target specific step edge profiles [28]. Consequently, the ROI parameters are not as numerous as compared to Canny detection, although still required to ensure isolated step edges with the desired features.

If there are less than three ROIs selected, the image is not considered suitable for deriving a representative mean MTF for that scene. The process is repeated for all images to obtain a representative performance measure for the given system.

The mean MTF is taken for each image in the database, and compared to the mean ISO 12233 measurements, averaged across the target edges from the entire field of view. Theoretically, the maximum MTF in the database should be near the optimum performance of the imaging system, derived from a perfect edge. Therefore, it should approximate the standard ISO 12233 measurement.

\section{Parameters}

The input parameters of the ISO 12233 algorithm are given in Table 1. The recommended dimensions of the ROI (which typically is manually selected) are no smaller than 64 pixels width by of $80-500$ pixels height. Variations in the edge angle ( 5 degrees commonly) result 


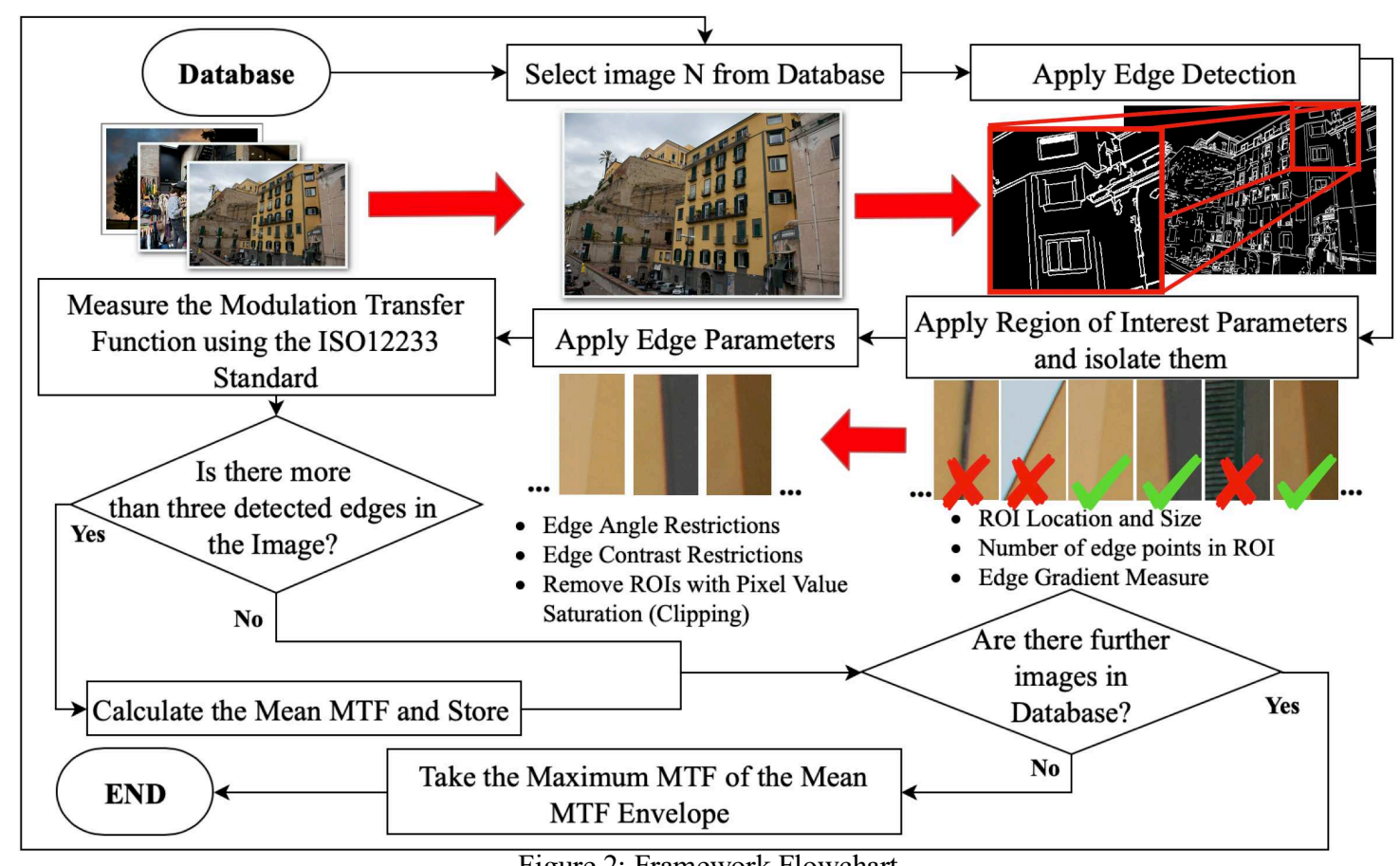

Figure 2: Framework Flowchart

in some variation in the measured MTF [29, 30, 31], the extent of which differs between studies; the acceptable edge angle range is not currently stated in the standard. The angle has to be smaller than 45 degrees, whereas the tolerable limit is around 30 degrees [29]. The contrast of the input step edge should be relatively low, below 10:1, the standard recommends it to be $4: 1$.

The initial parameters for our framework are listed in Table 1. Note, there are no edge contrast restrictions, and for a ROI to be valid there needs to be a minimum of 15 edge detection points within the area.

The selected ROI for these initial tests has a width of 27 pixels, 58\% smaller than the recommended 64 [29, 32] to allow for a maximum amount of step edges to be selected from the images. The trade-off is the precision in the measurements. To test this precision, simulated edges were used ranging in ROI width, from 7 pixels to over

\begin{tabular}{lll} 
Parameter & ISO 12233 & Set As \\
\hline ROI Size & $\begin{array}{l}>64 \times 80-500 \\
\text { pixels }\end{array}$ & $27 \times 41$ pixels \\
Angle & $<45 \mathrm{deg}$. & $2.5-42.5 \mathrm{deg}$. \\
Contrast & $\begin{array}{l}<10: 1, \\
4: 1 \mathrm{recommended}\end{array}$ & No contrast limit \\
$\begin{array}{ll}\text { Num. of } \\
\text { edge points } \\
\text { in ROI }\end{array}$ & $\mathrm{n} / \mathrm{a}$ & 15 \\
\hline & & \\
\hline
\end{tabular}

700 pixels, whilst the ROI height has been changed to maintain the same aspect ratio. Figure 3 shows the Mean Absolute Error (MAE) in the MTF measurement from the optimum. The green ' $\mathrm{X}$ ' indicates the recommended minimum 64 pixel width ROI, and the red ' $\mathrm{X}$ ' the ROI width used here, of 27 pixels. The test indicated a very small MAE (of only 0.0059) for the selected study ROI.

Further study is required to determine if this level of MAE is significant in the MTF measurement. In addition, studies on the effects of edge angle should be conducted to determine whether the angle range used in these initial studies is within the MTF measurement error limits.

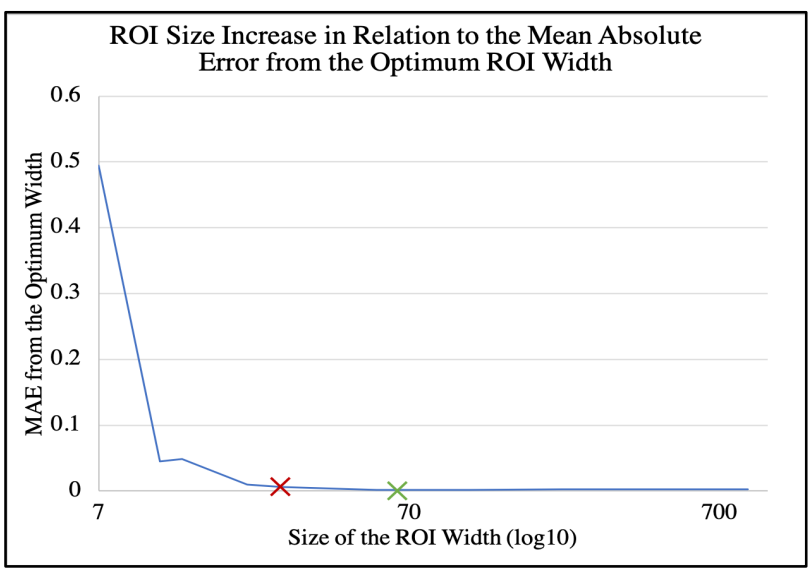

Figure 3: The variation caused by Region of Interest size.

\section{The Effects of Image Noise}

\subsection{Methodology}

To study the effects of noise on our proposed method, an artificial test image was created with a series of vertical edges, at angles ranging from 2.5 to 42.5 degrees. A hyperbolic tangent function was used to represent the step edge profiles.

Varying amounts of white Gaussian noise (signal 
independent) was added to the test image, resulting in signal-to-noise ratios (SNR), 5:1 (14dB), 10:1 (20dB), 20:1 (26dB), 40:1 (32dB), 50:1 (34dB). 27x41 pixel crops of the edges at these various noise levels are shown in Figure 4.

These six versions of the test image were put through the framework in Figure 1, and the responses were analyzed when using the Canny and the matched filtering edge detection techniques.

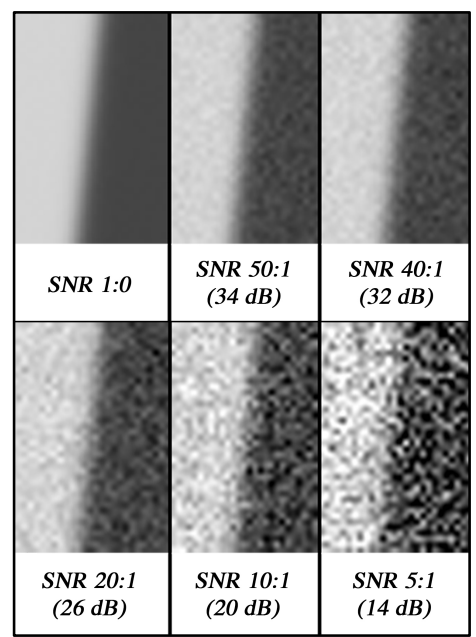

Figure 4: Table of the SNRs and a Chart Crop

\subsection{Results}

This study resulted in two main observations. The first is that the matched filter is not a suitable method for detecting noisy edges. It only detected edges at SNR 40:1 (low noise). However, by lowering the threshold of the matched, it appears that edges at the higher noise levels can be in fact detected, but at the expense of measurement accuracy. In contrast the Canny filter detected all edges at all noise levels, down to SNR 5:1 (high noise).

An issue with the current version of the framework is in the stage where specific step edge parameters are applied to the ROIs. Specifically, the stage where the gradient is taken to determine whether there is a step edge within the ROI, as mentioned in Section 3. At high noise levels, the noise level surpasses the floor noise set within the algorithm. As a result, more than one positive or negative gradients are found, therefore the algorithm doesn't validate this as a usable edge.

There are several possible solutions being investigated currently, including pre-evaluating the noise floor for each individual image, as well as using a variable noise reduction filter prior to taking the ROI gradient. There are advantages, and disadvantages of each of these that need to be considered. A disadvantage to these solutions may include allowing a ROI with more than one edge to pass through the system, if the second edge is below the noise floor. For the current tests, the parameter that restricts the noise floor was removed.

The second observation is on the variation introduced into the MTF measurement with the introduction of noise. A study conducted by Williams [29], showed that, for a $64 \times 128$ pixel ROI, a good MTF measurement can be obtained from an edge with a SNR down to 20:1. The results shown in Figures 5 and 6 agree with this finding, however the use of the smaller ROI introduced slightly more variation in the higher frequencies. Within the same study, Williams showed that by having the height of the ROI at 128 pixels, but using a narrower width of 18 pixels, the accuracy of the MTFs taken from targets with SNRs below 20:1 improved. In addition to this, by blurring either side of the ROI edge, without touching the edge spread, i.e. tail filtering, also helps to reduce the effects of image noise on the measured MTF [33].

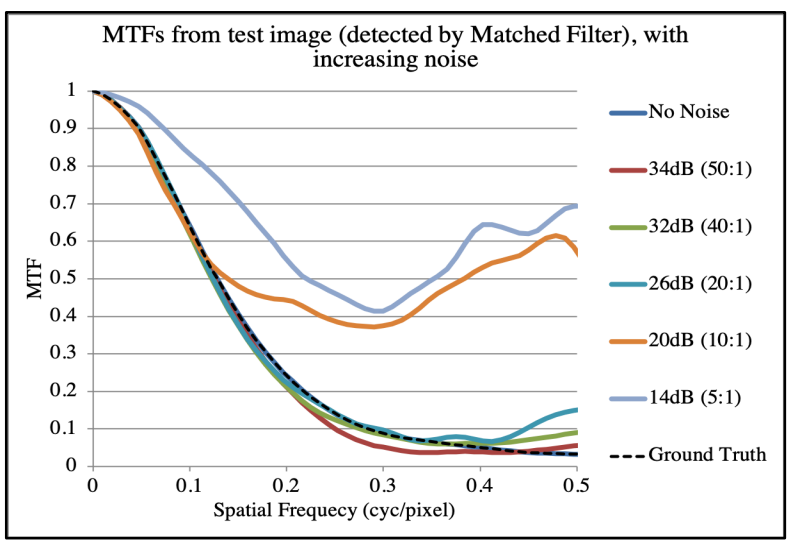

Figure 5: The variation introduced by noise when using the matched filter detection

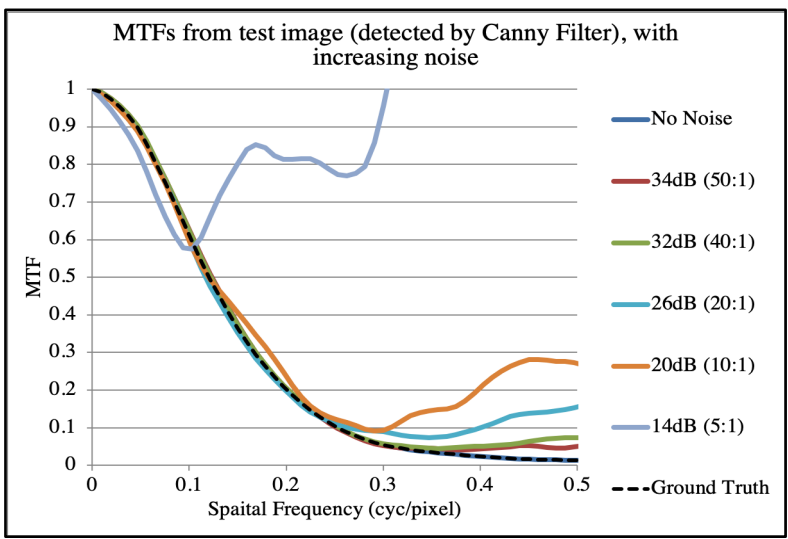

Figure 6: The variation introduced by noise when using the Canny detection

\section{Framework implementation}

\subsection{Methodology}

Implementation of the proposed framework was achieved using two small image datasets.

The first consisted of 50 images, all captured with a Nikon D800 DSLR and a Nikkor 24-70mm f/2.8G lens, set to $24 \mathrm{~mm}$ focal length at aperture $\mathrm{f} / 4$. It is important to note that, the pixel size of the imaging sensor, the lens optics, lens focus, and lens aperture setting are the main 


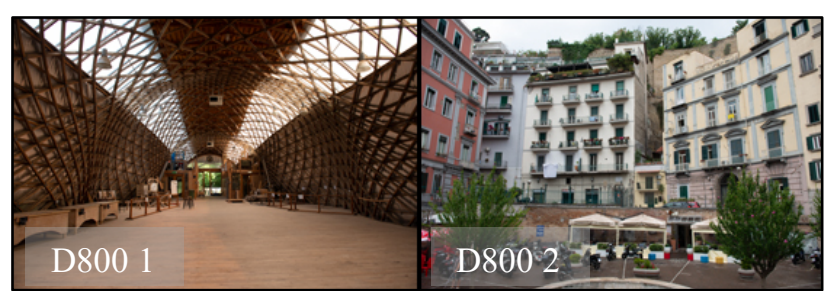

Figure 7: Two example images from the DSLR Image Database

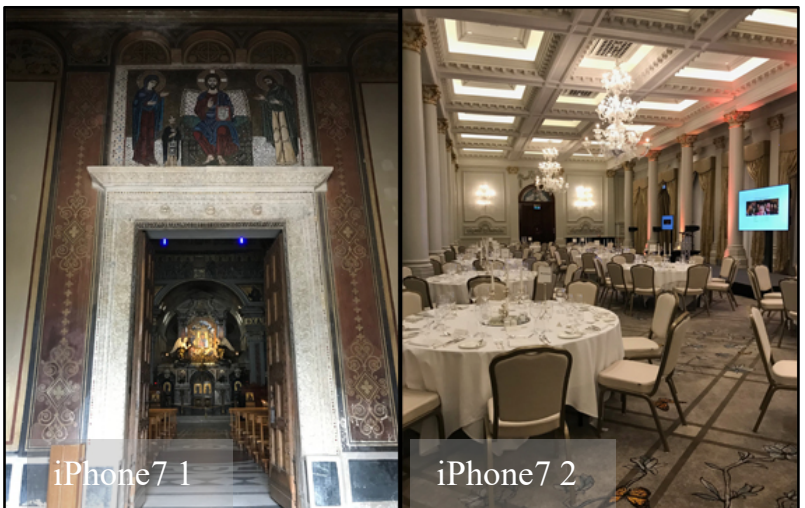

Figure 8: Two example images from the Smartphone Camera Image Database

factors affecting the camera MTF, thus in a meaningful performance study these need to be kept constant. All images in this dataset were TIFF files, converted from RAW, with sharpening and noise reduction turned off to minimize the amount of non-linear processing and saved in Adobe RGB color space.

The second dataset consisted of 87 images, captured with an Apple iPhone7. All images were JPEG files, directly outputted from the smartphone, saved in a variety of color spaces, but assumed here to be sRGB when the image data were linearized.

The images in both datasets were chosen so that they had a fair amount of well-lit edges, although the actual scene contents varied considerably. The majority of images consisted of urban and architectural contents; some rural and forest images were included too. Figures 7 and 8 show two examples from both databases.

It should be noted that for accurate measurement of the imaging systems' performance, more varied, larger datasets should be considered, but for the initial testing the employed datasets were deemed as sufficient.

\subsection{Individual image MTF measurements}

The MTF performance for both imaging systems were measured using the ISO 12233 methodology. The ROI size was set to the same size as for our algorithm for a direct comparison. The target edge contrast was $4: 1$ and a 5 degree angle ( \pm 3 degrees). The ROIs were detected using two methods: i) manual selection, ii) through the proposed algorithm. The manually selected measurement is considered as the systems' ground truth.

In Figures 9 and 10, the blue dashed curves correspond to mean MTFs, measured from the ISO test chart and by manually selecting the edges across the entire field of view. The green dashed curves indicate the same mean MTFs, when edges are detected using our algorithm. The orange dashed curves correspond to a single MTF measured from the chart at the center of the field of view (highest preforming area for a imaging system). Finally, the red dashed curve is the mean MTF for that particular MTF scene, or system envelope.

In Figure 9 the envelope of MTF curves is shown for the four example images (Figures 7 and 8). The edges for these curves were detected using the matched filter, although the same observations are made when using the Canny edge detector.

When comparing green and blue curves, we notice only a negligible variation between them, indicating that manually selected and automatically detected/processed edges deliver the same measurement, thus suggesting that the algorithm performs satisfactorily with the test chart. The slight variation (within MTF measurement error) is probably due to the number of edges being detected; the automated selection took all possible $27 \times 41$ pixel ROIs, whilst the manual selection took one ROI per chart edge.

Analyzing the two example DSLR images, the MTF envelope for the D800 1 (Figure 9 (a)) is low, below the mean ISO 12233 MTF. Whilst the D800 2 (Figure 9 (b)) envelope surrounds the mean ISO 12233 curve and does not go beyond the maximum preforming ISO 12233 measure, taken from the center of the field of view.

This result from the D800 2 image is an exact representation of what is theoretically expected, as discussed in Section 2. When detecting edges from the scene, an edge that corresponds to a perfect edge input will produce the maximum performance MTF curve, measured using the ISO12233 algorithm. Other step edges, i.e. edges within the MTF envelope, have the potential to describe the system/scene interactions further, i.e., performance variations due to edge field of view location, depth of field and system scene dependency.

System performance ranges across the field of view due to the optical characteristics of the lens, thus we expect a range of MTF performance curves from each scene. Looking at results from individual images, the D800 image 1 has 'usable' edges towards the corners of the field of view - the lowest performing areas of the system; this explains the low position of the measured MTF envelope. The D800 image 2 has 'usable' edges across the entire field of view, thus the envelope is broader, meaning that this image produces a more representative MTF measurement.

Similar observations can be made with the smartphone imaging system, Figures 9 (c) and (d). Both images have edges at the center of the scene, but they are too small to be processed. The 'usable' edges are located toward the border of the frame. As a result, the MTF envelopes are 


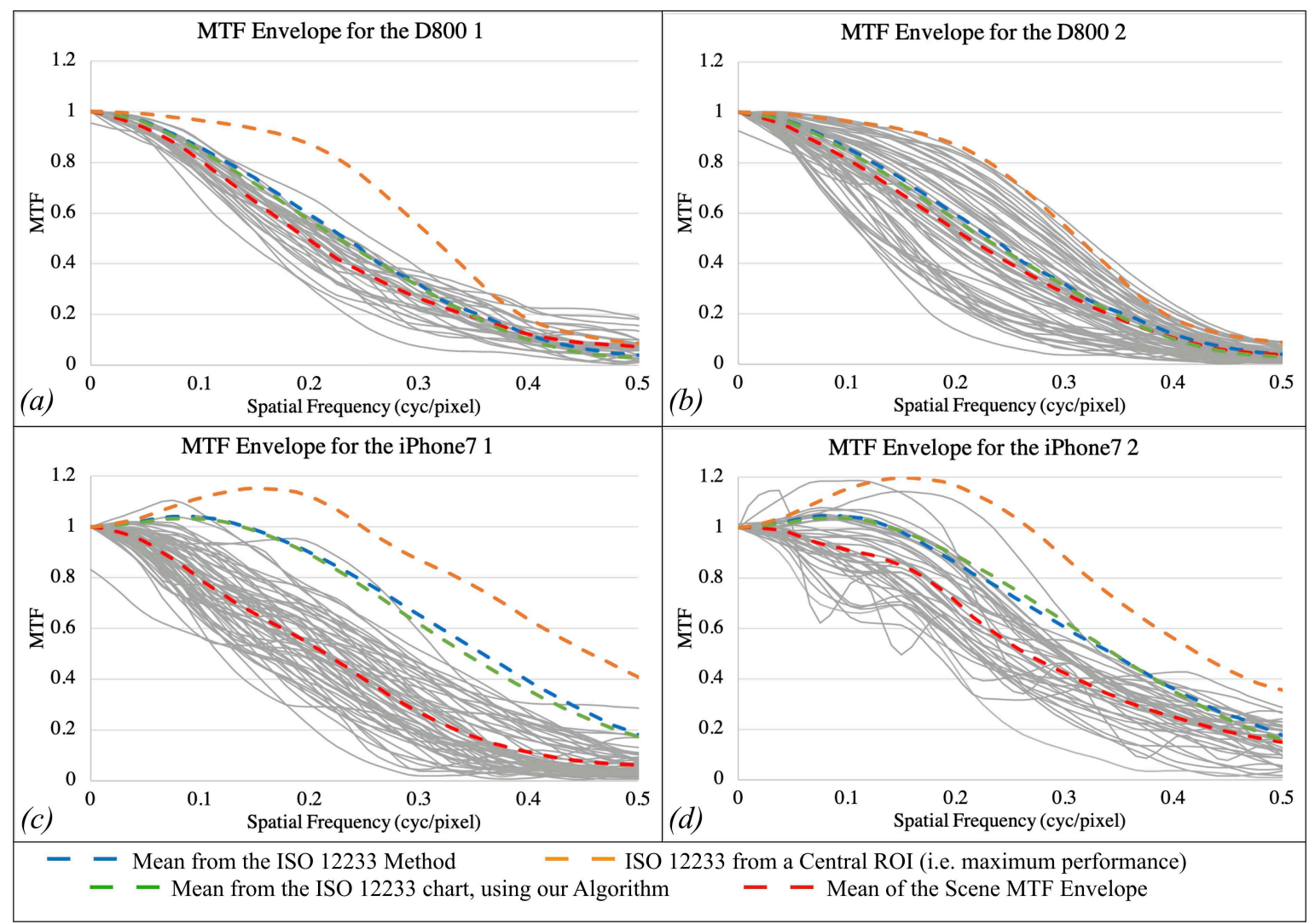

Figure 9: The MTF Envelopes, in a single edge orientation, for the four example images, where (a) and (b) from the Nikon D800 DSLR and (c) and (d) from the iPhone7 smartphone camera.

low, below the mean ISO 12233 curves. In addition, for a very non-linear system, where scene dependent image processing and compression is applied, there are further observations to be made.

The MTF curves have higher levels of noisy data. Thus, further parameters are needed to help reduce the selection of the corresponding edges.

Also, it is clear there is sharpening being applied to some edges that give the higher MTF curves, identified by the lobe $>1$ in the low frequencies. This characteristic becomes less prominent for the the weaker edges.

\subsection{Mean System MTF measurements}

As seen from Figure 2, the next step in the framework is the collection of all the mean images' MTFs to give a performance measure for the given imaging system database, as shown in Figure 10.

Comparison of results from the Canny edge detection, with those of the matched filter showed that the MTF spread from both methods vary little - thus only result from the former are shown here. The Canny detector identified more images in both datasets than the matched filter $(21 \%$ in the DSLR database, and $28 \%$ more in the smartphone database), but the results produced were slightly noisier.

For both DSLR and Smartphone systems, the mean system MTFs derived from the image datasets are lower than those measured with the ISO method (from the target). This is expected since all edges in the target are 'perfect' whilst this is not true for the images.

Further analysis is necessary to classify the image edges according to gradient, location within the depth of field, etc., for producing fully meaningful MTFs.

\section{Conclusions}

In summary, the proposed framework is an adaptation of the ISO 12233 Slanted-Edge MTF method: instead of using an image of a test chart taken in controlled conditions, suitable edges are derived from natural pictorial scenes. In this paper, both the Canny filter and a feature matched filter were used for this purpose of edge detection.

Such an approach has many advantages; it can derive system performance in real life (instead of laboratory) conditions. Also, it can describe better the performance of non-linear, scene dependent camera processes. Thus, such a performance measure can be a more representative input parameter to engineering image quality models than traditional MTFs. i.e. it is not only system dependent, but also scene dependent. It is important to note that the effect of non-linear sharpening is a current issue with the edge MTF measure as a whole. 




Figure 10: The images' mean MTF envelopes, in a single edge orientation, for each camera system database, detected using the Canny Edge Detection.

A scene dependent camera performance measure, unlike a test chart that includes "perfect edges" (with known spatial frequency content), will not produce a constant measure for the system. Natural scenes contain edges with varying spatial frequency content. The MTF produced is a relative measure for that particular image input. It may not give the system's maximum (true) performance, but will take into account other factors, such as focus, depth of field, edge location, non-linear processing etc. The long-term goal for this measure is to link scene content (using for example natural scene statistics) to derived MTFs, so that we end up with a consistent system performance measure.

Preliminary studies indicated that the proposed method is promising. The MTF envelopes produced from the natural scenes are closely related to results produced by the ISO 12233 algorithm, as long as there is a near to 'perfect edges' within in the scene. However, there are still many questions left unanswered and considerable work is still required to determine if such an approach is suitable for producing robust results.

Between the two edge detection methods, the results indicate that there is little difference in the mean measured MTFs. There are advantages and disadvantages in both methods.
The Canny edge detection is able to detect edges at high levels of image noise, picking up edges at lower than the currently minimum recommended SNRs [29]. The disadvantage of the Canny filter is that much further processing is needed to isolate suitable step edges.

The feature matched filter has the advantage of flagging only the edges that matched the template. The normalized 2D cross-correlation method, used in this study, also gives some leniency, a threshold that allows slight variation around the detection template. The most prominent disadvantage of the matched filter is its inability to detect edges with noise levels below SNR 40:1. A further disadvantage is that the templates used for the convolution kernels are essentially based on a simulated edge at various orientations. As a result, the edge profile may not necessarily be a match to the edge profiles within a specific image.

The results from this initial study have shown that, from each scene, a number of MTFs can be produced. These are located below the ISO 12233 MTF, derived from a perfect edge on a test chart, measured from the center of the field of view, i.e. the maximum preforming MTF for a given system. This produced range of MTFs is due to three main factors: the edge location within the camera field of view; the position of the edge within the depth of field of the image; and the scene dependent image processing and compression. Further research is required to separate the effects of these three factors.

Not all the 'suitable' edges within a scene are being used in measurements. Edges close to other edges and textures are excluded; the current ROI size is too large to isolate each one of them individually. But if a smaller ROI is used, error is introduced to the MTF measurement (Figure 3).

Noise is currently an issue with the proposed method, due to the chosen smaller ROI than the recommended. The resulting error is pronounced in the higher frequencies. In addition, the ROI processing does not take into account high floor noise levels automatically, thus edges with SNRs below 20:1 are not currently selected, even if flagged by the Canny filter.

Further studies will look into the ROI size, take into account the floor noise and investigate methods to help reduce the effects of noise, such as adding tail filtering, [29]. Future work should also consider other parameters relating to edge contrast.

Further steps would include testing with more extensive image datasets, containing images with broader variations in scene contents. Through investigation of natural scene statistics, the resulting scene MTFs envelopes will be classified under scene types, allowing the formation of a scene dependent MTF measure. In addition, though linking ROIs with derived MTFs we intend to identify statistically the system MTF.

We acknowledge NVIDIA Corporation with the donation of the Titan Xp GPU used for this research. 


\section{References}

[1] British Standard Institute, "BS ISO 12233:2017 Photography - Electronic still picture imaging Resolution and spatial frequency responses," BSI Stand. Publ., p. 62, 2017.

[2] British Standard Institute, "BS ISO 15739:2017 Photography — Electronic still-picture imaging Noise measurements," BSI Stand. Publ., p. 42, 2017.

[3] R. Branca, S. Triantaphillidou, and P. Burns,

"Texture MTF from images of natural scenes," Soc. Imaging Sci. Technol., pp. 113-120, 2017.

[4] E. W. S. Fry, S. Triantaphillidou, R. B. Jenkin, J. R. Jarvis, and R. E. Jacobson., "Validation of Modulation Transfer Functions and Noise Power Spectra from Natural Scenes," IEEE Trans. Image Process. - Rev.

[5] E. W. S. Fry, S. Triantaphillidou, R. E. Jacobson, J. R. Jarvis, and R. B. Jenkin, "Bridging the Gap Between Imaging Performance and Image Quality Measures," p. 6, 2018.

[6] S. J. Daly, "Visible differences predictor: an algorithm for the assessment of image fidelity," in Human Vision, Visual Processing, and Digital Display, 1993, vol. 3, pp. 179-206.

[7] Z. Wang, A. C. Bovik, H. R. Sheikh, S. Member, E. P. Simoncelli, and S. Member, "Image Quality Assessment : From Error Visibility to Structural Similarity," IEEE Trans. Image Process., vol. 13, no. 4, pp. 1-14, 2004.

[8] Z. Wang, E. P. Simoncelli, and A. C. Bovik, "MultiScale Structural Similarity For Image Quality Assessment," IEEE Asilomar Conf. Signals, vol. 2, pp. 1398-1402, 2003.

[9] R. Soundararajan and A. C. Bovik, "RRED indices: Reduced reference entropic differencing framework for image quality assessment," in 2011 IEEE International Conference on Acoustics, Speech and Signal Processing (ICASSP), 2011, pp. 1149-1152.

[10] Z. Wang and A. C. Bovik, "Reduced- and NoReference Image Quality Assessment," IEEE Signal Process. Mag., vol. 28, no. 6, pp. 29-40, 2011.

[11] Z. Wang and E. P. Simoncelli, "Reduced-reference image quality assessment using a wavelet-domain natural image statistic model," in Proc.SPIE, 2005, vol. 5666.

[12] X. Lv, M. Qin, X. Chen, and G. Wei, "No-reference image quality assessment based on statistics of convolution feature maps," AIP Conf. Proc., vol. 040034, pp. 1-6, 2018.

[13] C. Advisory and S. Association, IEEE Standard for Camera Phone Image Quality IEEE Standards Association Board of Governors. 2017.

[14] IEEE Standards Association Board of Governors, IEEE Standard for Camera Phone Image Quality. 2016.

[15] S. Triantaphillidou, J. Jarvis, A. Psarrou, and G. Gupta, "Contrast sensitivity in images of natural scenes, Signal Processing," Image Commun., vol. 75, pp. 64-75, 2019.
[16] P. G. J. Barten, "Evaluation of the Effect of Noise on Subjective Image Quality," Proc. SPIE 1453, Hum. Vision, Vis. Process. Digit. Disp. II, vol. 1453, pp. 2 $15,1991$.

[17] R. B. Jenkin, S. Triantaphillidou, and M. A. Richardson, "Effective Pictorial Information Capacity as an Image Quality Metric," Proc. SPIE 6494, Image Qual. Syst. Perform, vol. 4, 2007.

[18] K. Dabov, A. Foi, V. Katkovnik, and K. Egiazarian, "Image denoising with block-matching and 3D fi ltering," Proc SPIE 6064, Image Process Algorithms Syst Neural Networks, Mach Learn, pp. 1-12, 2006.

[19] Z. Mbarki, H. Seddik, S. Tebini, and E. Ben Braiek, "A new rapid auto-adapting diffusion function for adaptive anisotropic image de-noising and sharply conserved edges," Comput. Math. with Appl., vol. 74, no. 8, pp. 1751-1768, 2017.

[20] K. He, J. Sun, and X. Tang, "Guided Image Filtering," IEEE Trans Pattern Anal Mach Intell, pp. $1397-409,2010$

[21] C. R. Kumar and S. K. Srivatsa, "Enhancement of Image Sharpness with Bilateral and Adaptive Filter," vol. 6, no. 1, pp. 50-53, 2016.

[22] R. C. Sumner, R. Burada, I. Llc, and N. Kram, "The Effects of misregistration on the dead leaves crosscorrelation texture blur analysis," Electron. Imaging, Image Qual. Syst. Perform. XIV, pp. 121-129, 2017.

[23] R. Dainty, J. C.; Shaw, Image Science. Academic Press, 1992.

[24] R. Jenkin, "Noise, Sharpness, Resolution and Information," in The Manual of Photography, 10th ed., Burlington: Focal Press, 2011, pp. 433-456.

[25] P. D. Burns and D. Williams, "Refined slanted-edge measurement for practical camera and scanner testing," IS\&T PICS Conf., pp. 191-195, 2002.

[26] J. Canny, "A Computational Approach to Edge Detection," IEEE Trans. Pattern Anal. Mach. Intell., vol. PAMI-8, no. 6, pp. 679-698, 1986.

[27] R. M. Haralick and L. G. Shapiro, "Computer and Robot Vision," Volume II., Addison-Wesley, 1992, pp. 316-317.

[28] J. P. Lewis, "Fast Normalized Cross-Correlation," Vis. Interface, vol. 1995, no. 1, pp. 1-7, 1995.

[29] D. Williams, "Benchmarking of the ISO 12233 Slanted-edge Spatial Frequency Response Plug-in," IS\&T's PICS Conf., pp. 133-136, 1998.

[30] J. K. M. Roland, "A study of slanted-edge MTF stability and repeatability," SPIE Proc. Vol. 9396, pp. $1-9,2015$.

[31] M. Estribeau and P. Magnan, "Fast MTF measurement of CMOS imagers using ISO 12333 slanted-edge methodology," vol. 5570, p. 243, 2004.

[32] P. D. Burns and D. Williams, "Using Slanted Edge Analysis for Color Registration Measurement," Soc. Imaging Sci. Technol. Image Process. Image Qual. Image Capture, Syst. Conf., pp. 51-53, 1999.

[33] D. Williams and P. D. Burns, "Evolution of Slanted Edge Gradient SFR Measurement," Proc. SPIE 9016 - Image Qual. Syst. Perform. XI, vol. 9016, p. 8, 2014. 